\title{
Viabilidade de Vibrio cholerae 01 em diferentes tipos de águas em condições experimentais
}

\author{
Viability of Vibrio cholerae O1 in different types \\ of water under experimental conditions
}

Joseli Maria da Rocha Nogueira 1

Dália dos Prazeres Rodrigues 2

Ernesto Hofer 1,2

1 Laboratório de Zoonoses Bacterianas, Departamento de Bacteriologia, Instituto Oswaldo Cruz, Fundação Oswaldo Cruz. Av. Brasil 4365, Pavilhão Rocha Lima, Rio de Janeiro, RJ 21045-900, Brasil.

2 Centro de Referência Nacional de Cólera, Departamento

de Bacteriologia Instituto Oswaldo Cruz, Fundação Oswaldo Cruz. Av. Brasil 4365, Pavilhão Rocha Lima, Rio de Janeiro, $R J$ 21045-900, Brasil.

\begin{abstract}
The endemic and seasonal nature of cholera depends upon the survival of Vibrio cholerae $\mathrm{Ol}$ in a viable but not necessarily culturable state in ecological niches in aquatic environments during inter-epidemic periods, and investigation on the survival of this microorganism in such sites is therefore of the utmost importance. Weekly water aliquots were thus taken from 2 ponds and 2 rivers in the State of Rio de Janeiro. The samples were divided into two identical portions, one of which was autoclaved. A standardized dilution of V. cholerae O1 Inaba and of V. cholerae O1 Ogawa was inoculated in three aliquots of $100 \mathrm{ml}$ of these different water samples and maintained at different temperatures. Survival of the microorganisms in the aquatic environment under these different conditions was then analyzed. Regardless of the serotype, $\mathrm{V}$. cholerae serogroup 01 survived in water with salinity below $0.5 \%$ and at different temperatures for sufficient periods to spread through "bodies of water", demonstrating the need to constantly monitor areas of possible contamination, especially where the water is used for drinking, thus avoiding spread of the disease to surrounding populations.
\end{abstract}

Key words Vibrio cholerae; Survival; Aquatic Environment; Cholera

Resumo A natureza endêmica e sazonal da cólera depende da sobrevivência de Vibrio cholerae O1 em estado viável, mas não necessariamente cultivável em nichos ambientais aquáticos durante períodos interepidêmicos, sendo de suma importância o estudo da sobrevivência deste microrganismo nesses locais. Para tal, foram coletadas, semanalmente, alíquotas de água pertencentes a duas lagoas e dois rios do Estado do Rio de Janeiro. Esses volumes foram divididos em duas porções idênticas, uma das quais foi autoclavada. Uma diluição padronizada de V. cholerae O1 Inaba e de V. cholerae O1 Ogawa, foi inoculada em três alíquotas de 100ml dessas diferentes águas e mantidas em diferentes temperaturas. A sobrevivência desses microorganismos no âmbito aquático sob esses diferentes fatores foi então analisada. Os resultados demonstraram que o V. cholerae sorogrupo O1, independente do sorotipo, é capaz de se manter em água com salinidade abaixo de 0,5\% e em diferentes temperaturas, por períodos suficientes para sua disseminação através de "corpos d'água", demonstrando a necessidade de monitoramento constante em áreas de possível contaminação, principalmente onde a água é utilizada para o consumo, evitando assim, a disseminação da doença para as populações próximas a esses ambientes.

Palavras-chave Vibrio cholerae; Sobrevivência; Ambiente Aquático; Cólera 


\section{Introdução}

Na literatura, observa-se inúmeros relatos da ocorrência de Vibrio cholerae $\mathrm{O} 1 \mathrm{em}$ sistemas aquáticos (Feachem, 1981; Kaper et al., 1979; Wong et al., 1992).

Feachem (1981), Singleton et al. (1982a) e West (1989) sugerem a sobrevivência desses microrganismos por longos períodos em alguns desses ambientes, particularmente na água do mar.

Hoss \& Ness (1982) relatam sua presença em sedimento e água, independente das variações de natureza físico-química. Vimont \& Berche (2000) também demonstram sua presença em diferentes condições de $\mathrm{pH}$ e salinidade, permitindo, através dos resultados obtidos, a afirmação de que são membros autóctones na comunidade microbiana do estuário.

Colwell \& Spira (1993) sugerem que V. cholerae $\mathrm{O} 1$ possui habilidade de entrar em estado de latência em resposta à falta de nutrientes, a elevada salinidade e/ ou redução de temperatura. Em 1994, Cowell \& Huq demonstram que este estado, denominado viável mas não cultivável em meios de cultura convencionais, pode ser revertido. Nesses casos as células bacterianas reduzem de tamanho e tornam-se ovóides, não crescendo satisfatoriamente em meios de cultura comuns (Sanyal, 2000).

Há relatos de vários autores sobre a viabilidade de $V$. cholerae $\mathrm{O} 1$ em diferentes veículos aquosos (Blake et al., 1977; Sanyal, 2000; Sayamov \& Zaidenov, 1978).

Pérez-Rosas \& Hazen (1989), estudando água de rio propõe que $V$. cholerae $\mathrm{O} 1$ seja autóctone de águas tropicais. Por outro lado, Khan et al. (1984), estudando várias águas superficiais, na cidade de Dhaka, em Bangladesh, descrevem que essas não são eficientes reservatórios naturais desses microrganismos.

Miller et al. (1984) sugerem que ambientes com salinidade entre 0,25 e $3 \%$, tipicamente quentes, com temperaturas não inferiores a $5^{\circ} \mathrm{C}$ por períodos longos, são prováveis reservatórios naturais de $V$. cholerae $\mathrm{O} 1$ produtor de toxina colérica.

Borroto (1998) relaciona condições ecológicas com o favorecimento da sobrevivência em longo prazo do V. cholerae sugerindo que a altitude do meio aquático em estudo, em relação ao nível do mar, também é um fator importante para essas condições.

Huq et al. (1984), apoiados atualmente por Sanyal (2000), apontam o zooplâncton, principalmente os copépodes, como de grande importância na sobrevivência e multiplicação de V. cholerae no ambiente aquático.
Estudos laboratoriais têm demonstrado que V. cholerae $\mathrm{O} 1$ pode sobreviver por longos períodos de tempo em associação com algas e plantas aquáticas (Brown \& Sirenko, 1997; Islam et al., 1990; Sood et al., 1998; Tamplin et al., 1990).

Uchiyama (1998b), calculando isolamentos de V.cholerae em diferentes ambientes aquáticos, concluiu que este microorganismo ocorria muito mais em água de rio que no mar, sendo este ambiente mais propício a sua sobrevivência.

Deve-se ressaltar porém, que esses experimentos de sobrevivência devem ser analisados cuidadosamente pois, ambientes aquáticos como rios, lagos ou coleções de água, possuem sempre variação quanto à flora e fauna existentes. Estas condições não são reproduzíveis ou simuláveis em laboratório e, quando presente em seu habitat natural, V. cholerae $\mathrm{O} 1$ está em estreita associação com esses organismos (Furniss et al., 1984).

Em virtude do exposto e observando a discreta bibliografia nacional sobre o assunto, estabeleceu-se neste trabalho os seguintes objetivos:

a) Analisar a viabilidade de $V$. cholerae $\mathrm{O} 1 \mathrm{em}$ diferentes meios aquáticos;

b) Comparar a sobrevivência do sorotipos Inaba e Ogawa;

c) Avaliar o efeito de diferentes condições de temperatura na viabilidade desses microrganismos.

\section{Material e métodos}

\section{Amostragem}

Foram coletadas, de duas lagoas (Marapendi e Rodrigo de Freitas) e dois rios (Guandu e Sarapuí) localizados no Estado do Rio de Janeiro (Figura 1), semanalmente, volumes de $3.000 \mathrm{ml}$ de água, totalizando quatro amostras. Utilizouse frascos de boca larga, previamente esterilizados, sendo que o tempo decorrido do local da coleta até o processamento no laboratório não excedeu duas horas. A partir de cada coleta, foi determinado o pH da amostra, com auxílio de um potenciômetro e seu volume foi dividido em duas porções idênticas, uma das quais foi autoclavada a $121^{\circ} \mathrm{C}$ X $30^{\prime}$.

\section{Metodologia}

Em uma primeira etapa, para cada coleta, visando padronizar o inóculo ideal, foram efetuadas suspensões de $V$. cholerae Ol Inaba e Ogawa para obtenção, em volume final de $100 \mathrm{ml}$, das seguintes concentrações: $10^{4}, 105,10^{6}, 10^{7}$ e $10^{8}$ 


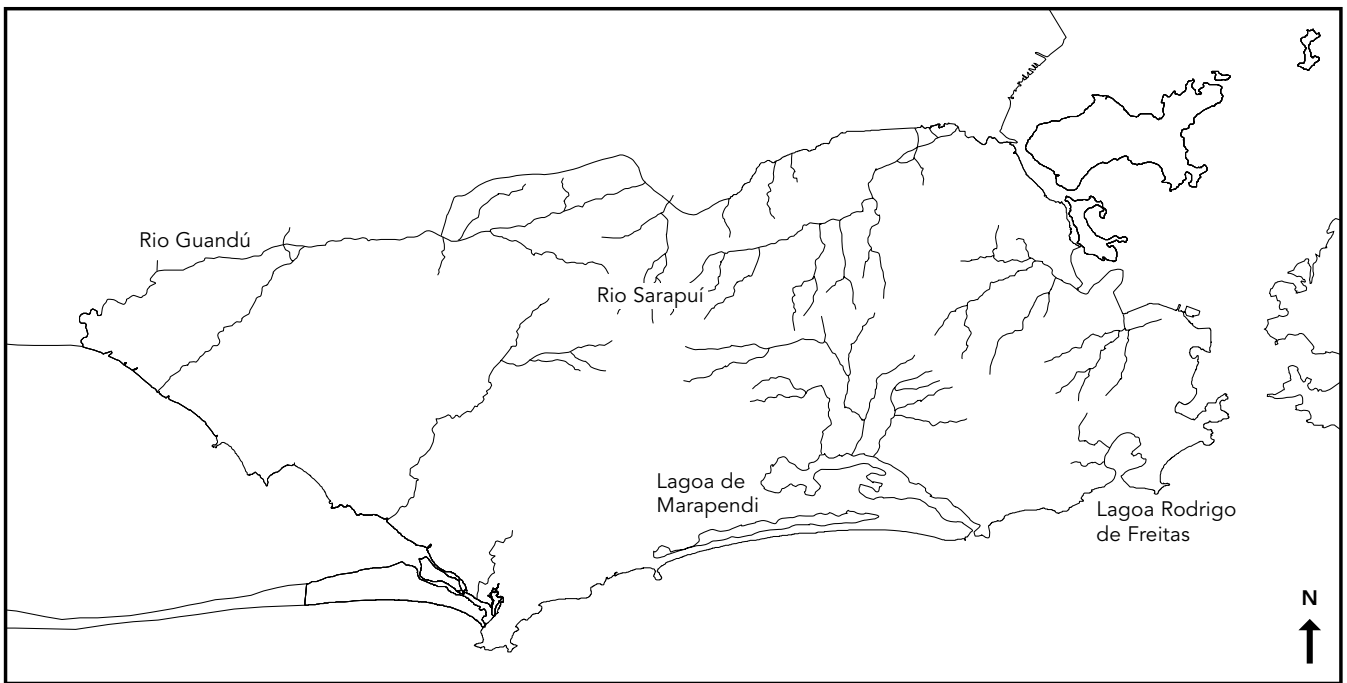

bactérias $/ \mathrm{ml}$. O inóculo ideal foi aquele capaz de permitir a contagem entre 30 e 300 Unidades Formadoras de Colônia (UFC) por placa (Furniss et al., 1984; Islam et al., 1990). Amostras clínicas toxigênicas de $V$. cholerae $\mathrm{O} 1$ Inaba e Ogawa (ATCC 14033 e ATCC 15748 respectivamente) foram semeadas em Ágar Nutriente com $1 \%$ de $\mathrm{NaCl}$. Após 18-24 horas, a 37C, o crescimento foi suspenso em solução salina 0,85\% e centrifugado a 3.000RPM por $15 \mathrm{minu}$ tos, repetindo-se esse procedimento por três vezes (Furniss et al., 1984; Islam et al., 1990).

Em seqüência, foram realizadas suspensões destas células lavadas, ajustadas com auxílio de um espectrofotômetro, para obtenção, em volume final de $100 \mathrm{ml}$, correspondentes a concentrações dos tubos 1 e 4 da Escala de Mac Farland. As diferentes concentrações, após homogeneização em vortex, foram plaqueadas, com auxílio de alça de Drigalski, em Ágar TCBS (Difco) e incubadas por 18-24 horas a $37{ }^{\circ} \mathrm{C}$. Após esse período, efetuou-se a contagem das UFC, selecionando assim, a diluição ideal (Furniss et al., 1984; Islam et al., 1990).

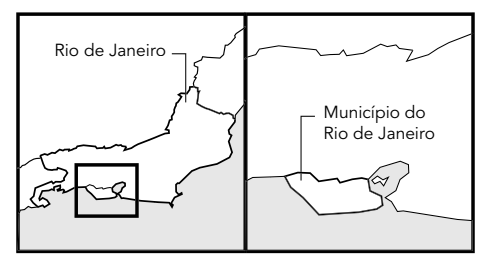

Em uma segunda etapa, a diluição ideal de $V$. cholerae $\mathrm{O} 1$ Inaba e de V. cholerae O1 Ogawa, foi inoculada em três alíquotas de $100 \mathrm{ml}$ da água coletada, as quais foram mantidas em temperatura ambiente, geladeira $(4 \circ \mathrm{C})$ e a $37 \circ \mathrm{C}$. Após intervalo de 24 horas, inóculos de $0,1 \mathrm{ml}$ foram semeados em Ágar TCBS, em triplicata. Essa operação foi repetida em intervalos de tempo de $2,4,7,14,21,28,45$ e 60 dias. Paralelamente, utilizamos o mesmo procedimento empregando as alíquotas autoclavadas (Chevalier \& Vandekerkove, 1980; Furniss et al., 1984).

Testou-se ainda, a manutenção da toxigenicidade das amostras inoculadas a cada intervalo de tempo usado para contagem, pelo processo de micro-aglutinação passiva reversa em látex (VET RPLA-OXOID).

\section{Resultados e discussão}

A possibilidade de propagação de $V$. cholerae O1 pela água, reconhecida há mais de um século, tem como aliada, o lançamento de dejetos 
urbanos em coleções de água, principalmente em grandes centros como é o caso do Rio de Janeiro. A pesquisa de sua viabilidade em condições experimentais, embora não reproduza fielmente as interações do habitat natural, propicia um modelo muito útil para possíveis comparações.

Os resultados deste estudo, estão apresentados na Figura 2, de acordo com as diferentes temperaturas utilizadas, onde se observa a manutenção dos sorotipos Inaba e Ogawa por aproximadamente o mesmo período, sendo que, nas amostras previamente esterilizadas houve recuperação de ambos os sorotipos até o 45o dia em temperatura ambiente e a 4 oC.

O maior tempo de sobrevivência em amostra não esterilizada (autoclavada), foi observado na água da Lagoa Rodrigo de Freitas mantida a $4 \circ \mathrm{C}$, na qual, $V$. cholerae $\mathrm{O} 1$ sorotipo Ogawa foi recuperado até o 21o dia. Entretanto, na água da Lagoa de Marapendi à mesma temperatura, este sorotipo somente foi recuperado até o sétimo dia, o mesmo ocorrendo com as águas dos rios Guandu e Sarapuí.

Ao compararmos os resultados da sobrevivência desse microrganismo em uma mesma amostra de água, (temperatura, salinidade, $\mathrm{pH}$ ) apenas eliminando sua competição, podemos observar a manutenção da sua sobrevivência por períodos extremamente maiores do que na água não estéril. Como essa água, foi esterilizada por autoclavação e não filtragem, houve a possibilidade da manutenção de nutrientes e possíveis interações com outros organismos, como por exemplo copépodes (Colwell \& Spira, 1993), que apesar de mortos podem ainda interagir com os víbrios. Estes achados corroboram com os de Uchiyama (1997) onde, em experimento semelhante, houve aumento do número de organismos iniciais em água de rio autoclavada, porém não houve recuperação após o 10o dia em água não autoclavada.

Nas amostras não autoclavadas, de ambas lagoas, recuperou-se, além das amostras previamente semeadas, outros integrantes da família Vibrionaceae, sendo que em todas as amostras colhidas (lagoas e rios) isolou-se grande número de outras bactérias.

Observou-se ainda a manutenção da toxigenicidade das amostras ATCC recuperadas.

Quanto ao fator temperatura, nossos resultados demonstraram prolongada manutenção a 4०C, se contrapondo aos de Uchiyama (1998a), onde $V$. cholerae não conseguiu ser mantido a $5^{\circ} \mathrm{C}$; este autor, sugere ao final de seu trabalho, que esses microrganismos conseguiriam sobreviver a essa temperatura na água de rio se estivessem aderidos a partículas de sedimento. O mesmo autor em outros estudos (Uchiyama, 1998b; Uchiyama et al., 1989) determina a salinidade como fator importante nestes casos, e embora muitos vibrios sejam halofílicos, aponta a água de rio mais propícia a sua manutenção que a água do mar, sendo que em salinidades inferiores, esses microorganismos poderiam se manter em temperaturas abaixo de $10 \circ \mathrm{C}$. Em nosso trabalho, não observamos essa afirmação, pois em ecossistemas de salinidade distinta (Lagoa de Marapendi - 22,5\%o e Rios Sarapuí/Guandu - 0,5\%o) tivemos sobrevivência até o 28o dia tanto em temperatura de geladeira como de ambiente, aparentemente independente desse fator. Na mesma linha de pensamento, porém enfocando a possível associação com os copépodos, Huq et al. (1984) sugerem em contraposição, que níveis mais altos de salinidade aliados a altas temperaturas e um $\mathrm{pH}$ alcalino favorecem o crescimento máximo de V. cholerae, embora em nosso trabalho não tenhamos observado esse conjunto de fatores aliados, pois o $\mathrm{pH}$ dos corpos d'água utilizados em nossos experimentos, em nenhuma das coletas mostrou-se alcalino. Outros trabalhos apontam estes fatores como benéficos para sua manutenção (Miller et al., 1984; Singleton et al., 1982b; Tamplin \& Colwell, 1986.), sugerindo que ambientes com salinidade entre $0,25 \mathrm{e}$ $3 \%$, tipicamente quentes, com temperaturas não inferiores a $5^{\circ} \mathrm{C}$ por períodos longos, são prováveis reservatórios naturais de $V$. cholerae O1 produtor de toxina colérica.

Nesse contexto, conclui-se que o V. cholerae sorogrupo $\mathrm{O} 1$, independente do sorotipo, é capaz de se manter em água com salinidade abaixo de $0,5 \%$ e em diferentes temperaturas por períodos suficientes para sua disseminação através de "corpos de água”, demonstrando a necessidade de monitoramento constante em áreas de possível contaminação, principalmente onde a água é utilizada para o consumo, evitando assim, a disseminação da doença para as populações próximas a esses ambientes. 
Sobrevivência em condições experimentais de V. cholerae O1 (Inaba e Ogawa) nos veículos aquosos, mantidos em diferentes temperaturas.
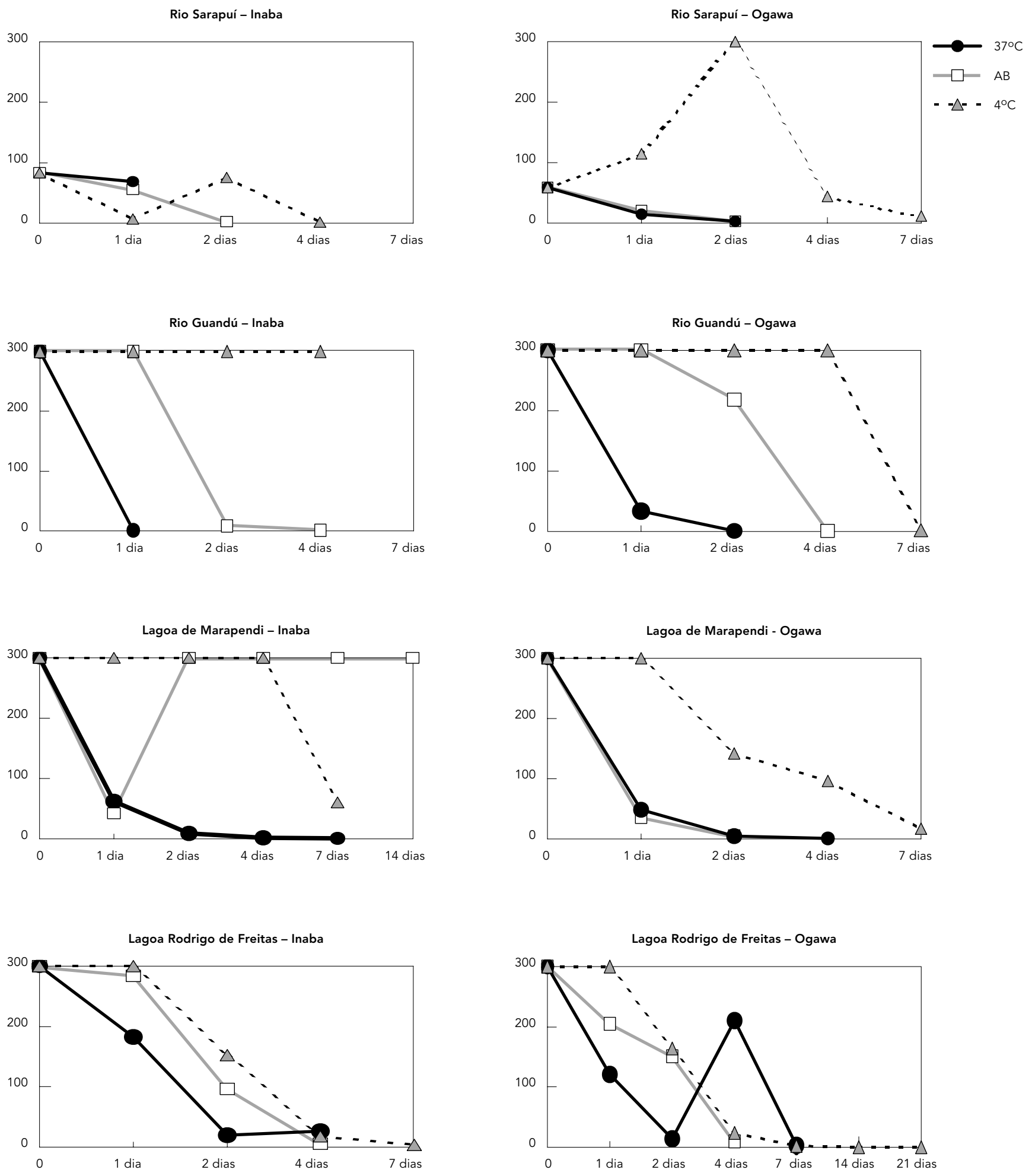


\section{Agradecimentos}

Nós agradecemos aos membros e técnicos do Centro de Referência Nacional de Cólera e outras enteroinfecções bacterianas e à Fundação Oswaldo Cruz (FIOCRUZ), pelo inestimável auxílio prático e disponibilidade das instalações. Agradecemos igualmente, ao apoio financeiro do Conselho Nacional para Desenvolvimento Científico e Tecnológico (CNPq).

\section{Referências}

BLAKE, P. A.; ROSENBERG, M. L.; FLORENCIA, J.; COSTA, J. B.; QUINTINO, L. D. P. \& GANGAROSA, E. J., 1977. Cholera in Portugal, 1974. II. Transmission by bottled mineral water. American Journal of Epidemiology, 105:344-348.

BORROTO, R. J., 1998. Survival of Vibrio cholerae O1 in freshwater surface and endemic cholera: A geological hypothesis. Revista Panamericana de Salud Pública, 4:371-374.

BROWN, I. I. \& SIRENKO, L. A., 1997. The role of the sodium cycle of energy coupling in the emergence and persistence of natural foci of modern cholera. Biochemistry (Moscow), 62:225-230.

CHEVALIER, M. \& VANDERKERKOVE, M., 1980. Experimental study on the survival of Vibrio cholerae (El-Tor biotype) in sea-water. Bulletin de la Société de Pathologie Exotique et de ses Filiales, 73: 364-372.

COLWELL, R. R. \& HUQ, A., 1994. Vibrios in the environment: Viable but nonculturable Vibrio cholerae. In: Vibrio Cholerae and Cholerae: Molecular to Global Perspectives (I. K. Wachsmuth, P. A. Blake \& Ø. Olsvik, ed.), pp. 117-133, Washington, DC: ASM Press.

COLWELL, R. R. \& SPIRA, W. M., 1993. The ecology of Vibrio cholerae. In: Cholera: Current Topics in Infections Disease (D. Barua \& W. B. Greenough III, ed.), pp. 3-60, New York: Plenum Medical Book Co.

FEACHEM, R. G. A., 1981. Environmental aspects of cholera epidemiology. I. A review of selected topics reports of endemic and epidemic situations during 1961-1980. Tropical Diseases Bulletin, 78: 675-698.

FURNISS, A. L.; LEE, J. V. \& DONOVAN, T. J., 1984. The Vibrios. Monograph Series 11. London: Her Majesty's Stationery Office.

HOSS, M. A. \& NESS, G. E., 1982. Survival of Vibrio cholerae and Escherichia coli in estuarine waters and sediments. Applied and Environmental Microbiology, 43:578-584.

HUQ, A.; WEST, P. A.; SMALL, E. B.; HUQ, M. I. \& COLWELL, R. R., 1984. Influence of water temperature, salinity, and $\mathrm{pH}$ on survival and growth of toxigenic Vibrio cholerae serovar $\mathrm{O} 1$ associated with live copepods in laboratory microcosms. $A p$ plied and Environmental Microbiology, 48:420424.
ISLAM, M. S.; DRASAR, B. S. \& BRADLEY, D. J., 1990. Long-term persistence of toxigenic Vibrio choler$a e \mathrm{O} 1$ in the mucilaginous sheath of blue-green alga, Anabaena variabilis. Journal of Tropical Medicine and Hygiene, 93:133-139.

KAPER, J.; LOCKMAN, H.; COLWELL, R. R. \& JOSEPH, S. W., 1979. Ecology, serology and enterotoxin production of Vibrio cholerae in Chesapeake Bay. Applied and Environmental Microbiology, 37:91103.

KHAN, M. U.; SHAHIDULLAH, M.; HAQUE, S. \& WASEQUE, U. A., 1984. Presence of vibrios in surface water and their relation with cholera in a community. Tropical and Geographical Medicine, 36:335-340.

MILLER, C. J.; DRASAR, B. S. \& FEACHEM, R. G., 1984. Response of toxigenic $V$. cholerae $\mathrm{O} 1$ to physicochemical stresses in aquatic environments. Jornal of Hygiene, 93:475-495.

PEREZ-ROSAS, N. \& HAZEN, T. C., 1989. In situ survival of Vibrio cholerae and Escherichia coli in a tropical rain forest watershed. Applied and Environmental Microbiology, 55:495-499.

SANYAL, S. C., 2000. Cholera in its present day scenario. Journal of the Indian Medical Association, 7:371-376.

SAYAMOV, R. M. \& ZAIDENOV, A. M., 1978. Survival and properties of cholera vibrios cultivated in mineral water. Zhurnal Mikrobiologii, Epidemiologii, i Immunobiologii, 2:66-70.

SINGLETON, F. L.; ATTWELL, R. W.; JANGI, M. S. \& COLWELL, R. R., 1982a. Influence of salinity and organic nutrient concentration on survival and growth of Vibrio cholerae in aquatic microcosms. Applied and Environmental Microbiology, 43: 1080-1085.

SINGLETON, F. L.; ATTWELL, R. W.; JANGI, M. S. \& COLWELL, R. R., 1982b. Effects of temperature and salinity on Vibrio cholerae growth. Applied and Environmental Microbiology, 44:1047-1058.

SOOD, S.; KAPIL, A.; DAS, B. K. \& SETH, P., 1998. The survival strategy of Vibrio cholerae. Tropical Gastroenterology, 19:96-97.

TAMPLIN, M. L.; GAUZENS, A. L.; HUQ, A.; SACK, D. A. \& COLWELL, R. R., 1990. Attachment of Vibrio cholerae Serogroup O1 to Zooplankton and Phytoplankton of Bangladesh Waters. Applied and 
Environmental Microbiology, 33:1977-1980.

TAMPLIN, M. L. \& COLWELL, R. R., 1986. Effects of microcosm salinity and organic substrate concentration on production of Vibrio cholerae enterotoxin. Applied and Environmental Microbiology, 52:297-301.

UCHIYAMA, H., 1997. Survival of Vibrio cholerae non$\mathrm{O} 1$ in freshwater river. Japanese Journal of Public Health, 44:547-557.

UCHIYAMA, H., 1998a. Survival of Vibrio cholerae non-O1 in river sediment during cold season. Journal of the Japanese Association for Infectious Diseases, 72:218-222.

UCHIYAMA, H., 1998b. A number of Vibrio cholerae non-O1 isolated from aquatic environments. Journal of the Japanese Association for Infectious Diseases, 72:720-726.
UCHIYAMA, H.; TODOROKI, T. \& MATSUI, S., 1989. Effects of salinity on the survival of non-O1 Vibrio cholerae under environments of low temperature. Kansenshogaku Zasshi, 63:138-144.

WEST, P. A., 1989. The human pathogenic vibrios - A public health update with environmental perspectives. Epidemiology and Infection, 103:1-34.

VIMONT, S. \& BERCHE, P., 2000. NhaA, an $\mathrm{Na}+/ \mathrm{H}+$ Antiporter Involved in Environmental Survival of Vibrio cholerae. Journal of Bacteriology, 182:29372944.

WONG, H.; TING, S. \& SHIEH, W., 1992. Incidence of toxigenic vibrios in foods avaliable in Taiwan. Journal of Applied Bacteriology, 73:197-202.

Recebido em 5 de julho de 2001

Versão final reapresentada em 3 de janeiro de 2002

Aprovado em 25 de março de 2002 